\title{
Impacto del índice de masa corporal en la presión arterial medida con esfigmomanómetro de mercurio en niños y adolescentes con diabetes mellitus tipo 1
}

\author{
Liuba M. Aguirre-Salas ${ }^{1}$, J. Jesús Pérez-Molina ${ }^{2,3 *}$, Salvador Fonseca-Reyes ${ }^{4}$, Jorge A. Becerra-Villa ${ }^{2}$ y \\ Mayra del C. Silva-Camarena ${ }^{1}$ \\ ${ }^{1}$ Servicio de Endocrinología, División de Pediatría, Nuevo Hospital Civil de Guadalajara Dr. Juan I. Menchaca; ${ }^{2}$ División de Pediatría, Nuevo Hospital \\ Civil de Guadalajara Dr. Juan I. Menchaca; ${ }^{3}$ Departamento de Clínicas de la Reproducción Humana, Crecimiento y Desarrollo Infantil, Centro \\ Universitario de Ciencias de la Salud, Universidad de Guadalajara; ${ }^{4}$ Instituto de Investigación Cardiovascular, Centro Universitario de Ciencias de \\ la Salud, Universidad de Guadalajara. Guadalajara, Jalisco, México
}

\section{Resumen}

Introducción: Los pacientes con diabetes mellitus tipo 1 (DM1) y sobrepeso tienen más riesgo de desarrollar cambios en la presión arterial (PA), y esto incrementa su morbilidad y mortalidad cardiovascular. En este estudio se determinó la relación entre la PA y el índice de masa corporal (IMC) y el promedio de las tres últimas mediciones de hemoglobina glucosilada (HbA1c) de pacientes con DM1. Métodos: Estudio transversal analítico en niños y adolescentes con DM1 con más de un año de evolución. Las variables dependientes fueron la PA sistólica y diastólica medidas con esfigmomanómetro y las variables independientes, IMC y promedio de las últimas tres mediciones de la HbA1c. Se utilizó regresión lineal múltiple con intervalo de confianza del 95\%. Resultados: Se estudiaron 75 pacientes con DM1. La mediana del tiempo de evolución de la DM1 fue de 3.5 años (mínimo 1 año-máximo 14.8 años), el IMC $19.5 \pm 3.1 \mathrm{~kg} / \mathrm{cm}^{2}$ y la HbA1c $8.3 \pm 2.4 \%$. De los 75 pacientes, 66 presentaron $P A<$ percentil 90 y 9 PA $\geq$ percentil 90 (12\%). Se construyeron dos modelos de regresión lineal múltiple, con PA sistólica y diastólica como variables dependientes. Las posibles variables predictoras fueron sugeridas por el contexto teórico y el análisis estadístico. El IMC expresado en puntuación zeta (zIMC) fue predictor para PA sistólical diastólica. Los modelos sugirieron que a cada incremento de unidad del zIMC corresponde un aumento de 5.1 y $3.6 \mathrm{mmHg}$ de PA sistólica y diastólica, respectivamente. Conclusiones: Se observó una correlación positiva de la PA sistólica y la diastólica con el zIMC.

Palabras clave: Diabetes mellitus tipo 1. Presión arterial. Índice de masa corporal. Hemoglobina glucosilada. Niños. Adolescentes.

Disponible en internet: 13-05-2019 Bol Med Hosp Infant Mex. 2019;76:126-133 www.bmhim.com 


\title{
The impact of body mass index on blood pressure measured with mercury sphygmomanometer in children and adolescents with type 1 diabetes mellitus
}

\begin{abstract}
Background: Patients with type 1 diabetes mellitus (T1DM) and overweight have more risk to develop changes in blood pressure that increase cardiovascular morbidity and mortality. In this study, the relationship between blood pressure (BP) with the body mass index (BMI) and the average of the last three measurements of glycated hemoglobin (HbA1c) in patients with T1DM was determined. Methods: A cross-sectional analytical study was conducted in children and adolescents with T1DM with over a year since diagnosis. The dependent variables were systolic and diastolic BP, measured with a mercury sphygmomanometer. The independent variables were BMI and average of the last three measurements of $\mathrm{HbA1}$. A linear regression with a 95\% confidence interval was used. Results: Seventy-five patients with T1DM were studied. The median of disease duration was 3.5 years (min 1-max 14.8 years), BMI $19.5 \pm 3.1 \mathrm{~kg} / \mathrm{cm}^{2}$ and $\mathrm{HbA1c} 8.3 \pm 2.4 \%$. Sixty-six patients showed BP $<$ percentile 90 and $9 \mathrm{BP} \geq$ percentile 90 (12\%). Two models of linear regression were constructed, with systolic and diastolic $B P$ as dependent variables. The possible predictor variables were suggested by theoretical context and statistical analysis. The predictive variable of high BP was zBMI (body mass index expressed in z-score) for systolic and diastolic BP. Also, the models suggested that for an increase of one unit of $z B M I$, corresponded a rise of 5.1 and $3.6 \mathrm{mmHg}$ in systolic and diastolic BP, respectively. Conclusions: A positive correlation between systolic and diastolic BP with zBMI was observed.
\end{abstract}

Key words: Type 1 diabetes mellitus. Blood pressure. Body mass index. Glycated hemoglobin. Children. Adolescents.

\section{Introducción}

Los pacientes con diabetes mellitus tipo 1 (DM1) desarrollan complicaciones crónicas que aumentan de forma importante la morbilidad y mortalidad cardiovascular: dos de las más frecuentes son la hipertensión arterial (HTA) y la enfermedad renal crónica ${ }^{1,2}$.

Debido a que el riesgo de HTA en el niño y adolescente es más del doble de lo habitual a mayor tiempo de evolución de la $\mathrm{DM}^{3}{ }^{3}$, la identificación de la presión arterial $(\mathrm{PA})$ mediante una técnica estricta de medición es un procedimiento obligado en la evaluación de esta alteración metabólica.

En el ambiente clínico pediátrico, y con mayor frecuencia en investigación, la medición automatizada de la PA ha venido sustituyendo la medición tradicional con la técnica auscultatoria mediante esfigmomanómetro de mercurio, principalmente por problemas de toxicidad con el mercurio y la mayor disponibilidad de dispositivos electrónicos que requieren menor entrenamiento para su uso. Sin embargo, las lecturas de PA obtenidas mediante dispositivos electrónicos, en general, son significativamente más altas que las registradas mediante la técnica auscultatoria, con el riesgo de sobreestimar el diagnóstico de HTA4. Además, se debe señalar que los valores de referencia para considerar la PA alta en niños y adolescentes se obtuvieron utilizando el método auscultatorio con esfigmomanómetro de mercurio ${ }^{5}$.

En los diferentes estudios en los que se ha investigado la relación entre DM1 y PA alta ${ }^{2,3,5}$, se han utilizado con mayor frecuencia los dispositivos electrónicos o aneroides, y ocasionalmente, esfigmomanómetros de mercurio ${ }^{4,6}$.

La asociación entre HTA y DM1 se ha explicado, en gran parte, por el mayor grado de adiposidad del individuo y el pobre control metabólico ${ }^{2-9}$. Lo anterior puede ser influenciado por factores locales y por la técnica de medición de la PA, por lo que el objetivo de este estudio fue determinar la relación entre la PA con el índice de masa corporal (IMC) y el promedio de las tres últimas mediciones de hemoglobina glucosilada (HbA1c) en pacientes pediátricos con DM1.

\section{Métodos}

\section{Población de estudio}

Se realizó un estudio transversal analítico ${ }^{10}$ en niños y adolescentes con DM1 del Servicio de Endocrinología Pediátrica en el Nuevo Hospital Civil de Guadalajara Dr. Juan I. Menchaca, de noviembre de 2014 a febrero de 2016. La investigación fue aprobada por los Comités de Ética y de Investigación de la institución y se obtuvo el consentimiento por escrito de padres o tutores.

Se incluyeron consecutivamente pacientes diabéticos con un mínimo de evolución de la enfermedad de un año. Se dividieron en dos grupos, uno con PA <percentil 90 y otro con $P A \geq$ percentil $90^{11}$. Se consideraron como "expuestos", los individuos que presentaron un valor de $\mathrm{HbA} 1 \mathrm{c}>7.5 \%$. 
Se calculó una muestra con error alfa de $0.05 \%$, error beta de $20 \%$, razón de sujetos con normotensión e hipertensión de 1, porcentaje de sujetos con DM1 con hipertensión y $\mathrm{HbA} 1 \mathrm{c}>7.5 \%$ del $44 \%$, porcentaje de sujetos con DM1 sin hipertensión y $\mathrm{HbA} 1 \mathrm{C} \geq 7.5 \%$ del $15 \%$. La prevalencia de exposición (HbA1c $\geq 7.5$ de 15 y $44 \%$ ) fue obtenida en los pacientes con DM1 atendidos en el servicio de Endocrinología de la institución sede. La razón de momios (RM) mínima que se puede detectar fue 4.4. El tamaño de muestra sugerido fue 76 sujetos.

No se incluyeron pacientes con enfermedades genéticas, HTA secundaria, en tratamiento con fármacos que modificaran la PA, con hábito tabáquico y que consumieran drogas ilícitas, además de los pacientes con datos incompletos o los que solicitaron su retiro del estudio.

\section{Variables}

Las variables dependientes fueron la PA sistólica (PAS) y la diastólica (PAD), expresadas en $\mathrm{mmHg}$ para edad, sexo y talla, además del diagnóstico de normotensión y prehipertensión ${ }^{11}$.

Las variables independientes fueron el control metabólico, identificado por el promedio de las últimas tres mediciones de HbA1c, y la adiposidad, mediante el IMC.

La DM1 se consideró presente cuando hubo dos o más determinaciones de glucemia en ayuno $\geq 126 \mathrm{mg} / \mathrm{dl}$ o glucemia tomada al azar $\geq 200 \mathrm{mg} / \mathrm{dl}$ con síntomas de hiperglucemia y valores de $\mathrm{HbA} 1 \mathrm{c} \geq 6.5 \% \%^{12}$, además de que los pacientes hubieran presentado un cuadro abrupto de cetosis y ausencia de acantosis nigricans como dato clínico de resistencia a la insulina.

El control metabólico se definió con el promedio de las últimas tres determinaciones de $\mathrm{HbA} 1 \mathrm{c}$, que fueron medidas con un intervalo de 4 meses. Se consideró como buen control glucémico $\leq 7.5 \%$ y mal control $>7.5 \%$ para fines del análisis estadístico.

El IMC se utilizó como una medida indirecta del grado de adiposidad, de acuerdo con lo establecido por la Organización Mundial de la Salud (OMS), ya que existe correlación entre este y el grado de adiposidad. Se clasificó como bajo ( $\leq 1$ desviación estándar [DE]); normal $( \pm 1 \mathrm{DE})$; sobrepeso (> $1 \mathrm{DE}$ pero $\leq 2 \mathrm{DE})$ y obesidad (> $2 \mathrm{DE})^{13}$.

\section{Procedimientos}

Previa explicación de la investigación a tutores y pacientes, se obtuvieron el consentimiento y el asentimiento informados. Los pacientes con DM1 que cumplieron con los criterios de inclusión acudieron a la entrevista directa para la recolección de la información y determinación de parámetros antropométricos con el método de Habicht ${ }^{13}$.

Los individuos fueron evaluados en el consultorio de la clínica de hipertensión por un observador entrenado específicamente en la toma correcta de la PA, de acuerdo con los criterios establecidos por organismos internacionales ${ }^{11,14}$. La misma persona midió tres veces la PA de los individuos en cada una de dos visitas, con un intervalo de dos a seis semanas entre ambas vistas. Las mediciones de PA fueron realizadas entre las 10:00 y las 13:00 horas con un esfigmomanómetro de mercurio modelo escritorio no abatible (Tycos ${ }^{\circledR}$ Instrument, North Carolina, USA). A cada niño se le explicó el procedimiento y se le indicó no hablar durante la medición. El paciente se mantuvo sentado, con la espalda apoyada y los pies colocados sobre una superficie plana, en un ambiente confortable y en reposo durante cinco minutos. El brazo estaba descubierto hasta el hombro y apoyado sobre una superficie horizontal a nivel del corazón. Previamente, se midió la circunferencia del brazo para cumplir con el requerimiento de utilizar el brazalete más apropiado, con ancho de $40 \%$ y longitud del 80 al $100 \%$, y se centró sobre la arteria braquial, sin ceñir exageradamente ni dejándolo demasiado holgado; el borde inferior estuvo tres $\mathrm{cm}$ por arriba de la fosa antecubital. Después, se procedió a determinar el nivel de inflación máxima mediante la maniobra de Osler, que consiste en palpar la arteria radial y concomitantemente inflar la cámara del brazalete $30 \mathrm{mmHg}$ por arriba del punto de la desaparición del pulso correspondiente a la PAS, para evitar dolor o incomodidad durante la medición. Se desinfló el brazalete, se colocó el estetoscopio sobre la arteria braquial de modo que toda la superficie estuviese en contacto con la piel, se infló la cámara al nivel predeterminado rápidamente y de forma sostenida, liberando el aire de la cámara a una velocidad de $2 \mathrm{mmHg}$ por latido cardíaco. No se reinfló el brazalete tras haber iniciado la desinflación, a fin de confirmar la PAS o PAD. Se consideró PAS cuando aparecieron dos latidos continuos (Korotkoff fase I) y PAD al punto donde desaparecieron los ruidos (Korotkoff fase V). Se escuchó durante $10 \mathrm{mmHg}$ más por debajo del último ruido para confirmar la desaparición de los ruidos.

\section{Análisis estadístico}

A las variables cuantitativas se les calculó promedio y desviación estándar y se compararon con la prueba 
t de Student para dos muestras independientes si la distribución fue normal. Cuando la distribución fue asimétrica, se determinó la mediana y se compararon con la prueba $U$ de Mann-Whitney. Las variables cualitativas se expresaron en proporciones y se compararon con la prueba $X^{2}$ o exacta de Fisher según fue necesario. La asociación de las variables cualitativas con $\mathrm{PA} \geq$ percentil 90 se calculó con razón de momios.

La relación entre PAS y PAD con IMC, el promedio de las últimas tres mediciones de HbA1c y tiempo de evolución se llevó a cabo con el análisis de regresión lineal múltiple. En todos los cálculos, el intervalo de confianza fue del 95\% (IC 95\%). Los análisis fueron realizados con el programa estadístico para ciencias sociales (SPSS Statistics for Macintosh, Versión 22.0. Armonk, NY: IBM Corp.)

\section{Resultados}

Se estudiaron 75 niños y adolescentes con DM1, con edad de $14.3 \pm 2$ años, (rango de 10 a 19 años), el 56\% de sexo masculino y una relación masculino/femenino de 1.3. El peso fue de $48.2 \pm 12.3 \mathrm{~kg}$ (rango de $25.6 \mathrm{a}$ $87.7 \mathrm{~kg}$ ) y el IMC fue $19.5 \pm 3.1 \mathrm{~kg} / \mathrm{cm}^{2}$ : peso bajo en el $13.3 \%$, peso normal en el $68 \%$ y sobrepeso en el $18.6 \%$. No se detectó ningún caso de obesidad. El tiempo de evolución de la DM1 fue de $4.6 \pm 3.6$ años, con mediana de 3.5 años, mínimo de 1 y máximo de 14.8 años. El periodo de evolución de la DM1 fue $<5$ años en el $66.7 \%$ y $\geq 5$ en el $33.3 \%$.

La dosis total de insulina por día fue de $49 \pm 18 \mathrm{Ul}$. Se determinó la HbA1c en 74 pacientes, con promedio del porcentaje de $8.3 \pm 2.4 \%$ : la $\mathrm{HbA} 1 \mathrm{c}$ fue $<6.5$ en el $12.2 \%, \geq 6.5$ a $<8$ en el $32.4 \%$, $\geq 8$ a $<10$ en el $29.7 \%$, $\geq 10$ a $<12$ en el $13.5 \%$ y $\geq 12$ en el $12.2 \%$.

El promedio de las últimas tres mediciones de $\mathrm{HbA} 1 \mathrm{c}$ fue $>7.5 \%$ en siete individuos con PA $\geq$ percentil $90(78 \%)$ y en 41 sujetos con PA < percentil 90 (62\%).

La distribución de la frecuencia de las variables cuantitativas fue de tipo normal, dado que los valores del error típico del índice de asimetría y el de curtosis fueron cercanos a $0.5 \mathrm{y}<1$, respectivamente.

Los niveles de PAS y PAD fueron $103.7 \pm 9.4 \mathrm{mmHg}$ y $68.8 \pm 7 \mathrm{mmHg}$, respectivamente. Sesenta y seis individuos mostraron $\mathrm{PA}<$ percentil 90 y nueve $\mathrm{PA} \geq$ percentil 90 (12\%). De estos, la PAS alta se observó en el $4 \%$, la PAD alta en el $6.6 \%$ y ambas elevadas en el $1.3 \%$.

Al contrastar las variables cuantitativas de pacientes con $\mathrm{PA} \geq$ percentil 90 y con PA normal, los promedios para edad, peso al nacer, peso actual, tiempo de evolución, dosis total de insulina y HbA1c fueron semejantes en ambos grupos de estudio. Por otra parte, los promedios del IMC y de circunferencia de cintura fueron mayores en el grupo de pacientes con $\mathrm{PA} \geq$ percentil 90 (Tabla 1).

De la comparación de las variables cualitativas, se encontró que el antecedente de HTA y obesidad en la familia, ser pretérmino al nacer, que la madre haya aumentado $>15 \mathrm{~kg}$ en su embarazo, porcentaje de evolución $\geq 5$ años y HbA1c $>7.5 \%$ se distribuyeron de manera semejante en los dos grupos de estudio.

Además, el sexo femenino y los índices de cintura e IMC elevados fueron más frecuentes en pacientes con prehipertensión (Tabla 2).

Para analizar la relación de las variables cuantitativas con la variable PA, se construyeron dos modelos de regresión lineal múltiple, que tuvieron como variables dependientes PAS y PAD. Las posibles variables predictoras fueron las que el contexto teórico y el análisis estadístico previo sugirieron. Después de comprobar el ajuste de los modelos (resumen de ANOVA con relación lineal significativa, $p<0.001$ ) y residuos independientes entre sí (estadístico Durbin-Watson 1.94 para PA sistólica y 2.1 para PA diastólica y media de los residuos de cero), la única variable que fue predictora de PA $\geq$ percentil 90 fue el IMC, tanto para PAS $(\beta=0.54 ; p<0.001)$ como para $\operatorname{PAD}(\beta=0.51 ; p<0.001)$.

Los modelos de regresión también sugirieron que, por cada incremento de una unidad de zIMC (IMC expresado en puntuación zeta), correspondía un aumento de $5.1 \mathrm{mmHg}$ de PAS (Tabla 3) y $3.02 \mathrm{mmHg}$ de PAD (Tabla 4).

\section{Discusión}

Los niños y adolescentes con DM1 presentan un mayor riesgo de complicaciones vasculares y renales durante la evolución de su enfermedad metabólica ${ }^{8}$. Gröber-Grätz, et al..$^{15}$ encontraron una mayor prevalencia de otros factores de riesgo como el sobrepeso y la obesidad, que pueden asociarse a mayor morbilidad cardiovascular en niños sin diabetes. Flores-Huerta, et al. ${ }^{16}$ documentaron también una asociación positiva del sobrepeso y obesidad con los niveles de PA (obtenida con diferentes técnicas de medición) en niños y adolescentes $\sin$ diabetes, en los que observaron que, a mayor IMC, la PA tanto sistólica como diastólica fue más alta. Sin embargo, en ninguno de estos trabajos se realizó análisis de correlación entre IMC con PAS y PAD. Además, con el método conocido como medición ambulatoria de la PA (MAPA) de 24 horas, se ha demostrado 
Tabla 1. Características de los individuos con diabetes mellitus tipo 1 con PA $\geq$ P90 y con PA $<$ P90

\begin{tabular}{|c|c|c|c|c|c|}
\hline \multirow[t]{2}{*}{ Variable } & \multicolumn{2}{|c|}{$\begin{array}{c}P A \geq P 90 \\
(n=9)\end{array}$} & \multicolumn{2}{|c|}{$\begin{array}{c}P A<P 90 \\
(n=66)\end{array}$} & \multirow[t]{2}{*}{$\mathbf{p}^{*}$} \\
\hline & Promedio & DE & Promedio & DE & \\
\hline Edad (años) & 14.3 & 1.9 & 14.3 & 2.1 & 0.946 \\
\hline Peso al nacer $(\mathrm{g})$ & 3077 & 493 & 3183 & 672 & 0.651 \\
\hline Peso $(\mathrm{kg})^{* *}$ & 58.6 & $34-88$ & 50.1 & $25-80$ & 0.451 \\
\hline IMC $\left(\mathrm{kg} / \mathrm{m}^{2}\right)$ & 22.3 & 2.9 & 19.7 & 3.1 & 0.022 \\
\hline Circunferencia cintura $(\mathrm{cm})$ & 74.8 & 8.1 & 68.7 & 6.6 & 0.013 \\
\hline Tiempo de evolución (años) ${ }^{* *}$ & 7.2 & $1-12$ & 3.4 & $1-15$ & 0.451 \\
\hline Dosis total de insulina por día (UI) & 56.0 & 17.7 & 48.2 & 18.5 & 0.239 \\
\hline Dosis de insulina por kg de peso por día (UI) & 1.0 & 0.3 & 0.9 & 0.3 & 0.576 \\
\hline $\mathrm{HbA1c}(\%)$ & 9.7 & 2.3 & 8.7 & 2.4 & 0.265 \\
\hline PAS (mmHg) & 112.7 & 11.9 & 102.5 & 8.4 & 0.002 \\
\hline PAD (mmHg) & 75.6 & 9.9 & 67.9 & 6.1 & 0.002 \\
\hline
\end{tabular}

* Valor de $\mathrm{p}$ al comparar las medias por la prueba t de Student o las medianas con prueba U de Mann-Whitney para dos muestras independientes.

**Variables con distribución asimétrica: mediana (mín-máx).

DE: desviación estándar; IMC: índice de masa corporal; HbA1c: hemoglobina glucosilada; PA: presión arterial; PAD: presión arterial diastólica; PAS: presión arteria sistólica; P90: percentil 90; UI: unidades internacionales.

Tabla 2. Antecedentes de los individuos con diabetes mellitus tipo 1 con PA $\geq$ P90 y con PA $<$ P90

\begin{tabular}{|c|c|c|c|c|c|c|}
\hline \multirow[t]{2}{*}{ Variable } & \multicolumn{2}{|c|}{$\mathbf{P A} \geq \mathbf{P 9 0}$} & \multicolumn{2}{|c|}{$\mathbf{P A}<\mathbf{P 9 0}$} & \multirow[t]{2}{*}{ RM (IC95\%) } & \multirow[t]{2}{*}{$\mathbf{p}$} \\
\hline & $n / N$ & $\%$ & $n / N$ & $\%$ & & \\
\hline Sexo femenino & $7 / 9$ & 77.7 & $26 / 66$ & 39.9 & $5.4(1.1-27.9)$ & 0.037 \\
\hline Antecedente de hipertensos en la familia & $5 / 9$ & 55.5 & $35 / 66$ & 53.1 & $1.1(0.3-4.5)$ & 0.443 \\
\hline Antecedente de obesidad en la familia & $6 / 9$ & 66.6 & $42 / 66$ & 63.6 & $1.1(0.3-4.9)$ & 0.586 \\
\hline Ser pretérmino al nacer & $1 / 9$ & 11.1 & $8 / 66$ & 12.1 & $0.9(0.1-8.2)$ & 0.705 \\
\hline Aumento de peso $>15 \mathrm{~kg}$ en su gestación & $2 / 9$ & 22.2 & $21 / 64^{*}$ & 32.8 & $0.6(0.1-3.1)$ & 0.261 \\
\hline Evolución de la DM1 >3.5 años & $6 / 9$ & 66.6 & $31 / 66$ & 46.9 & $2.2(0.5-9.8)$ & 0.453 \\
\hline Sobrepeso por adiposidad & $4 / 9$ & 44.4 & $10 / 66$ & 15.1 & $4.5(1.0-19.6)$ & 0.017 \\
\hline Índice de cintura ( $\geq 0.5$ vs. $<0.5)$ & $2 / 9$ & 22.2 & $4 / 66$ & 6.1 & $4.4(0.7-28.7)$ & 0.046 \\
\hline HbA1c $>7.5 \%$ (promedio de las tres últimas mediciones) & $7 / 9$ & 78.0 & $41 / 66$ & 62.0 & $2.1(0.4-11.1)$ & 0.600 \\
\hline
\end{tabular}

*Faltó información de algunos individuos de estudio.

DM1: diabetes mellitus tipo 1; IC: intervalo de confianza; HbA1c: hemoglobina glucosilada; PA: presión arterial; P90: percentil 90; RM: razón de momios. $\mathrm{p}$ : valor de $\mathrm{p}$ al comparar las proporciones con la prueba $\chi^{2}$ o prueba exacta de Fisher.

mayor asociación y predicción de daño cardiovascular comparado con la medición en consultorio ${ }^{17}$.

El niño y adolescente con diabetes y mayor grado de adiposidad, aparte de un frecuente descontrol glucémico, posee un alto riesgo de presentar niveles más altos de PA y, por consecuencia, mayor riesgo cardiovascular $^{18}$. Aunque existen estudios que abordan esta relación en diferentes poblaciones étnicas de niños con diabetes en algunas regiones del mundo ${ }^{2-3,17}$, no se conoce el efecto de una mayor adiposidad sobre el 
Tabla 3. Regresión lineal múltiple con presión arterial sistólica como variable dependiente

\begin{tabular}{|c|c|c|c|c|c|}
\hline \multirow[t]{2}{*}{ Variables predictoras } & \multicolumn{2}{|c|}{ Coeficientes no estandarizados } & \multirow{2}{*}{$\frac{\text { Coeficientes tipificados }}{\text { Beta }}$} & \multirow[t]{2}{*}{ IC $95 \%$} & \multirow[t]{2}{*}{$\mathbf{p}$} \\
\hline & B & Error típico & & & \\
\hline Constante & 103.262 & 3.183 & & $96.91-109.61$ & $<0.001$ \\
\hline zIMC & 5.086 & 1.037 & 0.538 & $3.02-7.15$ & $<0.001$ \\
\hline Sexo femenino & 1.631 & 1.980 & -0.86 & $-5.58-2.31$ & 0.413 \\
\hline Tiempo de evolución & 3.389 & 2,098 & -0.180 & $-7.57-0.797$ & 0.111 \\
\hline $\mathrm{HbA1c}$ & -2.268 & 2.042 & -0.116 & $-6.34-1.80$ & 0.270 \\
\hline Dosis de insulina por $\mathrm{kg}$ de peso & 4.573 & 3.019 & 0.169 & $-1.45-10.60$ & 0.134 \\
\hline
\end{tabular}

ANOVA del modelo. Significación $\leq 0.001 ; R^{2}$ corregida del modelo $=0.218$. Tiempo de evolución de la diabetes mellitus: $>3.5$ años. A cada incremento en una unidad de zIMC le corresponde un aumento de $5.086 \mathrm{mmHg}$ de presión arterial sistólica.

HbA1c: promedio de las tres últimas mediciones de hemoglobina glucosilada; IC: intervalo de confianza; zIMC: índice de masa corporal expresado en puntuación zeta.

Tabla 4. Regresión lineal múltiple con presión arterial diastólica como variable dependiente

\begin{tabular}{|c|c|c|c|c|c|}
\hline \multicolumn{6}{|c|}{ Presión arterial diastólica como variable dependiente } \\
\hline \multirow[t]{2}{*}{ Variables predictoras } & \multicolumn{2}{|c|}{ Coeficientes no estandarizados } & \multirow{2}{*}{$\begin{array}{c}\text { Coeficientes tipificados } \\
\text { Beta }\end{array}$} & \multirow[t]{2}{*}{ IC $95 \%$} & \multirow[t]{2}{*}{$\mathbf{p}$} \\
\hline & B & Error típico & & & \\
\hline Constante & 68.381 & 2.391 & & $63.61-73.15$ & $<0.001$ \\
\hline zIMC & 3.597 & 0.779 & 0.511 & $2.04-5.15$ & $<0.001$ \\
\hline Sexo femenino & -0.854 & 1.487 & -0.061 & $-3.82-2.1$ & 0.568 \\
\hline Tiempo de evolución & 0.170 & 1.576 & 0.012 & $-2.97-3.31$ & 0.914 \\
\hline $\mathrm{HbA1c}$ & -0.356 & 1.534 & -0.024 & $-3.42-2.70$ & 0.817 \\
\hline Dosis de insulina por $\mathrm{kg}$ de peso & 1.041 & 2.268 & 0.052 & $-3.48-5.56$ & 0.648 \\
\hline
\end{tabular}

ANOVA del modelo. Significación $\leq 0.001 ; R^{2}$ corregida del modelo $=0.204$. Tiempo de evolución de la diabetes mellitus: $>3.5$ años. A cada incremento en una unidad de zIMC le corresponde un aumento de $3.6 \mathrm{mmHg}$ de presión arterial diastólica.

$\mathrm{HbA1c}$ : promedio de las tres últimas mediciones de hemoglobina glucosilada; IC: intervalo de confianza; zIMC: índice de masa corporal expresado en puntuación zeta.

nivel de PA en la población mexicana mestiza u otras poblaciones de América Latina.

En este estudio se utilizó el IMC como una medida de estimación indirecta del grado de adiposidad, ya que se ha demostrado que es un buen marcador de grasa corporal, así como de su relación con la $\mathrm{PA}^{19}$. Los resultados obtenidos mostraron la relación del IMC con la PAS $(\beta=0.54)$ y con la PAD $(\beta=0.51)$, y que por cada incremento de una unidad de puntuación de zIMC en los niños con diabetes, correspondió un aumento de 5.1 de $\mathrm{mmHg}$ en la PA sistólica y de 3.6 de $\mathrm{mmHg}$ en la PA diastólica. Después de evaluar 164 pacientes con DM1 en Polonia, a quienes se les midió la PA con un dispositivo electrónico, Pietrzak, et al. ${ }^{3}$ también encontraron una correlación entre el IMC y la PA sistólica ( $\beta=0.37$ ) y diastólica ( $\beta=0.28$ ).

Otros autores, como Guimaraes et al. ${ }^{6}$ en Brasil, quienes midieron la PA con un dispositivo de mercurio y una técnica estricta de medición en una muestra grande de 536 niños y adolescentes no diabéticos, y Van Vliet, et al. ${ }^{8}$ en Holanda, en 283 niños con DM1, encontraron una frecuencia de PAS y PAD elevadas en sujetos con IMC alto. Desafortunadamente, no realizaron un análisis de correlación. Cerca del $40 \%$ de los sujetos de este último estudio presentaron sobrepeso u obesidad y mayor frecuencia de prehipertensión. En contraparte, de Oliveira, et al. ${ }^{2}$ en Brasil, en una muestra pequeña de 60 adolescentes con DM1 a quienes 
se les midió la PA en el consultorio con un dispositivo electrónico validado, no encontraron correlación significativa entre el aumento del IMC y la PA; no obstante, se observó una tendencia estadística entre el sobrepeso y una mayor PAD.

En el presente trabajo, se encontró el $12 \%$ de individuos con $\mathrm{PA} \geq$ percentil 90 , pero ninguno con $\mathrm{PA} \geq$ percentil 95 , muy probablemente por el tamaño reducido de la muestra, ya que Margeirsdottir, et al. ${ }^{1}$ en Noruega, después de estudiar una población de 1,658 pacientes con DM1, encontraron $\mathrm{PA} \geq$ percentil 95 en el $4 \%$ de los niños evaluados y un porcentaje con PA $\geq$ percentil 90 pero menor a PA < percentil 95 muy similar a la frecuencia del presente estudio.

Se han identificado diferentes mecanismos biológicos como explicación para esta relación entre mayor IMC y PA más elevada: a mayor cantidad de tejido adiposo, la resistencia a la insulina se incrementa y se liberan más citocinas inflamatorias, con el consecuente aumento de estrés oxidativo. Además, se activa el sistema renina-angiotensina-aldosterona, aumenta la disfunción vascular y la retención de sodio, y se observa una mayor actividad del sistema nervioso simpático en conjunto con una mayor disfunción renal, por lo general más intensa en presencia de albuminuria y que coadyuva a aumentar el riesgo cardiovascular ${ }^{20}$.

Otra variable relacionada con mayor nivel de PA en niños con DM1 ha sido el pobre control glucémico. En este sentido, de Oliveira, et al. ${ }^{2}$ observaron en niños y adolescentes con descontrol glucémico importante (HbA1c 11.6\%) niveles más altos de PA sistólica y diastólica, así como una correlación positiva con la PA diastólica, con un incremento de $1.73 \mathrm{mmHg}$ por cada $1 \%$ de aumento en la HbA1c.

En un estudio de tipo retrospectivo, Torchinsky, et al. ${ }^{21}$ evaluaron a 148 niños con DM1 en EE.UU. y encontraron un mayor promedio de PAD y frecuencia cardíaca a medida que aumentaron los niveles promedio de HbA1c. Incluso se observó una correlación con las dosis de insulina. En otro estudio en 106 niños norteamericanos con DM1, en el que se midió la presión por medio de MAPA con análisis bivariado, Chatterjee, et al. ${ }^{7}$ también encontraron mayor frecuencia en el descontrol glucémico en sujetos con prehipertensión e hipertensión. En contraste, Pietrzak, et al. ${ }^{3}$ no demostraron una correlación positiva entre HbA1c y PA mediante regresión lineal.

En relación con lo anterior, en este estudio no se encontró correlación entre el control glucémico y la PA, aunque en el análisis bivariado hubo una mayor frecuencia de $\mathrm{HbA} 1 \mathrm{c}$ alta en los sujetos con prehipertensión, a pesar de que no fue estadísticamente significativa. Se debe señalar que el promedio de los porcentajes de $\mathrm{HbA1c}$ de este estudio fue similar al reportado por otros autores ${ }^{3,7}$.

En cuanto al tiempo de evolución de la DM1, no se observó una relación con la PA. Sin embargo, Pietrzak, et al. ${ }^{3}$ sí documentaron una correlación positiva de este con la PAD. Además, de Oliveira, et al. ${ }^{2}$ reportaron que, a mayor tiempo de evolución de la DM1, mayor frecuencia de prehipertensión, únicamente en el análisis bivariado.

Cabe mencionar que los individuos con $\mathrm{PA} \geq$ percentil 90 contaron con mayor tiempo de evolución de la enfermedad, mayores niveles de HbA1c y mayor proporción de personas con inadecuado control glucémico, pero a pesar de ser valores clínicamente significativos, no mostraron significación estadística. Del mismo modo, la ganancia de peso mayor a $15 \mathrm{~kg}$ en la gestación no fue relevante.

Una limitación del presente estudio fue el tamaño reducido de la muestra, ya que con una prevalencia del $12 \%$ de PA $\geq$ percentil 90 , asumiendo las prevalencias de exposición a HbA1c del $15 \%$ y $44 \%$, el poder del estudio es cercano al $60 \%$ para identificar una RM de 4.5. Sin embargo, en esta investigación, la RM de la exposición a $\mathrm{HbA} 1 \mathrm{c}$ del $15 \%$ y $44 \%$ fue cercana a 2 y el poder del $20 \%$. A pesar de esto, el tamaño de la muestra fue suficiente para probar la relación entre el IMC y la PA. Otra limitación sería que en el estudio se estimó el grado de adiposidad a través del IMC y no con otras formas de evaluación. En relación con lo anterior, la OMS sugiere que se puede estimar el grado de adiposidad con el IMC, ya que entre ellos existe adecuada correlación ${ }^{13}$.

Por lo comentado anteriormente, se concluye que en este estudio se encontró una correlación positiva entre la PA sistólica y diastólica con el zIMC, semejante a la reportada en otros estudios de niños con DM1. No se encontró correlación entre la PA con el promedio de las tres últimas mediciones de $\mathrm{HbA} 1 \mathrm{c}$, probablemente debido al reducido tamaño de la muestra.

Por lo anterior, es importante lograr un equilibrio entre el ingreso y el gasto de energía en la práctica clínica, a través de un adecuado plan de alimentación, actividad física y uso adecuado de insulina, para evitar la ganancia exagerada de adiposidad, así como el seguimiento y tamizaje de complicaciones macrovasculares y microvasculares en pacientes con DM1, de acuerdo con las guías ISPAD $2018^{22}$. 


\section{Responsabilidades éticas}

Protección de personas y animales. Los autores declaran que para esta investigación no se han realizado experimentos en seres humanos ni en animales.

Confidencialidad de los datos. Los autores declaran que han seguido los protocolos de su centro de trabajo sobre la publicación de datos de pacientes.

Derecho a la privacidad y consentimiento informado. Los autores han obtenido el consentimiento informado de los pacientes o individuos referidos en el artículo. Este documento obra en poder del autor de correspondencia.

\section{Conflicto de intereses}

Los autores declaran que no existe ningún conflicto de intereses.

\section{Financiamiento}

Ninguno.

\section{Bibliografía}

1. Margeirsdottir HD, Larsen JR, Brunborg C, Overby NC, Dahl-Jørgensen K, Norwegian Study Group for Childhood Diabetes. High prevalence of cardiovascular risk factors in children and adolescents with type 1 diabetes: a population-based study. Diabetologia. 2008;51:554-61.

2. De Oliveira S, da Cunha Nascimento D, Tibana RA, de Oliveira SL, de Sousa Neto IV, Falleiros Maciel RK, et al. Elevated glycated hemoglobin levels impair blood pressure in children and adolescents with type 1 diabetes mellitus. Diabetol Metab Syndr. 2016;8:4

3. Pietrzak I, Mianowska B, Gadzicka A, Młynarski W, Szadkowska A. Blood pressure in children and adolescents with type 1 diabetes mellitus-the influence of body mass index and fat mass. Pediatr Endocrinol Diabetes Metab. 2009;15:240-5.

4. Fonseca-Reyes S, Romero-Velarde E, Torres-Gudiño E, Illescas-Zárate D, Forsyth-MacQuarrie AM. Comparison of auscultatory and oscillometric BP measurements in children with obesity and their effect on the diagnosis of arterial hypertension. Arch Cardiol Mex. 2018;88:16-24.

5. Rohani F, Hooman N, Moradi S, Mobarra M, Najafizadeh M, Tatarpoor $\mathrm{P}$. The prevalence of pre-hypertension in children with type 1 diabetes mellitus. Int J Prev Med. 2014;5:S44-9.

6. Guimarães IC, de Almeida AM, Santos AS, Barbosa DB, Guimarães AC. Blood pressure: effect of body mass index and of waist circumference on adolescents. Arq Bras Cardiol. 2008;90:393-9.
7. Chatterjee M, Speiser PW, Pellizzarri M, Carey DE, Fort P, Kreitzer PM, et al. Poor glycemic control is associated with abnormal changes in 24hour ambulatory blood pressure in children and adolescents with type 1 diabetes mellitus. J Pediatr Endocrinol Metab. 2009;22:1061-7.

8. van Vliet M, Van der Heyden JC, Diamant M, Von Rosenstiel IA, Schindhelm RK, Aanstoot HJ, et al. Overweight is highly prevalent in children with type 1 diabetes and associates with cardiometabolic risk. J Pediatr. 2010;156:923-9.

9. Knerr I, Dost A, Lepler R, Raile K, Schober E, Rascher W, et al. Diabetes Data Acquisition System for Prospective Surveillance (DPV) Scientific Initiative Germany and Austria. Tracking and prediction of arterial blood pressure from childhood to young adulthood in 868 patients with type 1 diabetes: a multicenter longitudinal survey in Germany and Austria. Diabetes Care. 2008;31:726-7.

10. Hernández B, Velasco-Mondragón HE. Encuestas transversales. Salud Publica Mex. 2000;42:447-55.

11. National High Blood Pressure Education Program Working Group on High Blood Pressure in Children and Adolescents. The fourth report on the diagnosis, evaluation, and treatment of high blood pressure in children and adolescents. Pediatrics. 2004;114:555-76.

12. Casallas Osorio JA. La Guía Completa y Actualizada para el diagnóstico, tratamiento y prevención de las complicaciones de la Diabetes Mellitus. ADA (American Diabetes Association) [Internet]; 2013. Disponible en: https://www.intramed.net/userfiles/2013/file/diabetes_2013.pdf.

13. World Health Organization. Measuring change in nutritional status. Guidelines for assessing of nutritional impact of supplementary feeding programs for vulnerable groups. Geneva: WHO; 1983.

14. Lurbe E, Cifkova R, Cruickshank JK, Dillon MJ, Ferreira I, Invitti C, European Society of Hypertension, et al. Management of high blood pressure in children and adolescents: recommendations of the European Society of Hypertension. J Hypertens. 2009;27:1719-42.

15. Gröber-Grätz D, Widhalm K, de Zwaan M, Reinehr T, Blüher S, Schwab KO, et al. Body mass index or waist circumference: which is the better predictor for hypertension and dyslipidemia in overweight/obese children and adolescents? Association of cardiovascular risk related to body mass index or waist circumference. Horm Res Paediatr. 2013; 80:170-8.

16. Flores-Huerta S, Klünder-Klünder M, Reyes de la Cruz L, Santos Jl. Increase in body mass index and waist circumference is associated with high blood pressure in children and adolescents in Mexico City. Arch Med Res. 2009;40:208-15.

17. Kotsis V, Stabouli S, Bouldin M, Low A, Toumanidis S, Zakopoulos N. Impact of obesity on 24-hour ambulatory blood pressure and hypertension. Hypertension. 2005;45:602-7.

18. Díaz-Cárdenas $C$, Wong $C$, Vargas-Catalán NA. Grado de control metabólico en niños y adolescentes con diabetes mellitus tipo 1. Rev Chil Pediatr. 2016;87:43-7.

19. Romero-Velarde E, Vásquez-Garibay EM, Álvarez-Román YA, Fonseca-Reyes S, Casillas-Toral E, Troyo-Sanromán R. Circunferencia de cintura y su asociación con factores de riesgo cardiovascular en niños y adolescentes con obesidad. Bol Med Hosp Infant Mex. 2013;70:358-63.

20. Cheung BM, Li C. Diabetes and hypertension: is there a common metabolic pathway? Curr Atheroscler Rep. 2012;14:160-6.

21. Torchinsky MY, Gomez R, Rao J, Vargas A, Mercante DE, Chalew SA. Poor glycemic control is associated with increased diastolic blood pressure and heart rate in children with Type 1 diabetes. J Diabetes Complications. 2004;18:220-3.

22. Donaghue KC, Marcovecchio ML, Wadwa RP, et al. ISPAD Clinical Practice Consensus Guidelines 2018: Microvascular and macrovascular complications in children and adolescents. Pediatr Diabetes. 2018;19:262-74. 